\title{
Laboreal
}

Volume $14 \mathrm{~N}^{\circ} 2$ | 2018

Digitalização e evolução do trabalho real

\section{Prácticas desarrolladas por los trabajadores con predominio del trabajo manual: caso de estiba de productos pesqueros}

Práticas desenvolvidas pelos trabalhadores em contexto de trabalho manual: o caso da estiva de produtos pesqueiros

Pratiques élaborées par les travailleurs exerçant une activité à prédominance manuelle: le cas de l'arrimage de produits de la pêche

Practices developed by workers who perform mainly manual work: the case of stowage of fish products

\section{Carolina Ullilen-Marcilla y Romel Ullilen-Marcilla}

\section{OpenEdition}

Edición electrónica

URL: http://journals.openedition.org/laboreal/685

DOI: $10.4000 /$ laboreal. 685

ISSN: 1646-5237

Editor

Universidade do Porto

Referencia electrónica

Carolina Ullilen-Marcilla y Romel Ullilen-Marcilla, « Prácticas desarrolladas por los trabajadores con predominio del trabajo manual: caso de estiba de productos pesqueros », Laboreal [En línea], Volume 14 N² | 2018, Publicado el 31 diciembre 2018, consultado el 06 octubre 2019. URL : http:// journals.openedition.org/laboreal/685; DOI : 10.4000/laboreal.685

Este documento fue generado automáticamente el 6 octubre 2019.

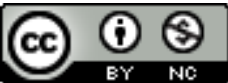

Laboreal está licenciado com uma Licença Creative Commons - Atribuição-NãoComercial 4.0 Internacional. 


\section{Prácticas desarrolladas por los trabajadores con predominio del trabajo manual: caso de estiba de productos pesqueros}

Práticas desenvolvidas pelos trabalhadores em contexto de trabalho manual: o caso da estiva de produtos pesqueiros

Pratiques élaborées par les travailleurs exerçant une activité à prédominance manuelle: le cas de l'arrimage de produits de la pêche

Practices developed by workers who perform mainly manual work: the case of stowage of fish products

Carolina Ullilen-Marcilla y Romel Ullilen-Marcilla

\section{NOTA DEL EDITOR}

http://dx.doi.org/10.15667/laborealxiv218cum

Manuscrito recibido em: agosto/2018

Aceite após peritagem: novembro/2018

\section{Introducción}

1 Para conservar la calidad de los productos pesqueros durante el transporte marítimo, los estibadores colocan los pescados congelados dentro de conteneros refrigeradores Reefer ubicados en los muelles de los terminales portuarios del principal puerto del Perú, actividad que es realizada manualmente en ambientes de bajas temperaturas. Uno de los problemas de salud a los cuales se exponen los estibadores son los trastornos músculo-esqueléticos (TME) por la manipulación permanente de cargas pesadas. 
2 En el Perú, la ley $N^{\circ} 27866$ considera la actividad del Trabajador Portuario como actividad de riesgo. Según el Decreto Supremo N003-2003-TR, el Estibador es reconocido dentro de las especialidades de los Trabajadores Portuarios, realizando todas las tareas, labores y funciones ordinarias del trabajo portuario en las operaciones de carga o estiba, descarga o desestiba y movilización de carga.

Es importante tener en cuenta que muchas empresas peruanas promueven la política de cero accidentes. Sin embargo, en el caso de las enfermedades profesionales de progresión lenta, muchos son los trabajadores que presentan dolencias e incluso aún no

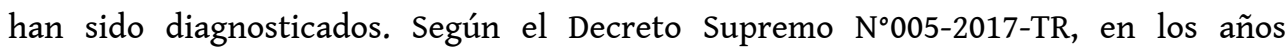
2011-2015, se notificaron 182 casos provocados por posturas forzadas y movimientos repetidos en el trabajo y 114 casos por lumbago, pudiendo aumentar estas cifras de manera significativa debido al sub-registro de enfermedades profesionales.

4 La política laboral en la mayoría de empresas está más orientada a evitar los accidentes de trabajo y cumplir un rol principalmente asistencial frente a las lesiones. Cada vez más son las empresas que tienen una población adulta, cuyas actividades laborales demandaban y/o siguen demandando altas exigencias físicas y mentales. Por lo tanto, arrastran un pasivo que significa un elevado costo a la organización.

5 Dada la escasez de estudios sobre la manipulación de productos pesqueros en el Perú y aparentemente en América Latina, la presente intervención desarrollada en una empresa de la industria marítima busca aportar a la comprensión de cómo se desarrolla la actividad de estiba de pescados congelados dentro de los contenedores en situación real bajo un enfoque sistémico y plantear medidas adaptadas a la realidad de la empresa, involucrando a los estibadores quienes han adquirido destrezas y habilidades.

6 Este trabajo comprende ocho secciones. En la segunda sección, se aborda el marco teórico, en la tercera sección, se plantea el objetivo de la intervención ergonómica, en la cuarta sección, se abarca la metodología. En la quinta sección, se presenta el análisis de la demanda de la empresa en estudio, en la sexta sección, se presentan los resultados, en la séptima y octava sección, se señalan las discusiones y conclusiones.

\section{Marco teórico}

7 Los TME son uno de los problemas más frecuentes de salud en los diferentes sectores productivos con un alto impacto en la calidad de vida y el desempeño laboral. El estudio de cohorte de Garg et al. (2014) realizado a 258 trabajadores muestra la influencia de los factores estresantes de carga en el riesgo del dolor lumbar, relacionándolo con el índice de levantamiento por el Instituto Nacional de Seguridad y Salud Ocupacional de los EE.UU. (NIOSH). Por otro lado, la exposición profesional al frío (natural o artificial) puede conllevar a la hipotermia y la congelación, además de los TME, estando relacionado el impacto del frio en función de la edad, genero, morfología / antropometría, fatiga, insuficiencia en la ingesta de alimentos y líquidos, ropa inadecuada, entre otros (Monroy \& Luna, 2015).

8 Para la prevención de los TME de origen laboral, resulta necesario tener en cuenta el enfoque multicausal para comprender su aparición, siendo el factor biomecánico el más estudiado y hacia el cual las empresas dirigen sus esfuerzos principalmente al realizar modificaciones al puesto de trabajo, los cuales son insuficiente para solucionar los problemas al no considerar los factores psicosociales y organizacionales (Bourgeois et 
al., 2006). Por lo tanto, es importante considerar la relación entre los TME y los riesgos psicosociales, puesto que el análisis del gesto profesional (movimiento humano al cual se le atribuye un significado) no se reduce a lo que pasa en las articulaciones, por lo que es necesario salir de una visión reducida del gesto centrándose en las alteraciones biomecánicas y acceder a una visión más global, integrando los determinantes psicosociales (Bourgeois \& De Gasparo, 2011).

9 En el plano de los TME, la presencia de diferentes factores de riesgo resulta de la combinación de una serie de determinantes (o causas) de origen técnico, organizacional y humano, es así, que el análisis de las situaciones de trabajo debe permitir poner en relación los determinantes de la actividad de trabajo (incluyendo las regulaciones del personal) y sus efectos tanto para el trabajador como para la empresa, por lo tanto, la prevención de los TME debe direccionarse sobre los determinantes de los factores de riesgo en la búsqueda de pistas de transformación (Bellemare et al., 2002.). No hay prevención de los TME sin pasar por la comprensión de la actividad por parte de aquellos que lo realizan, pasando de un enfoque clásico de la higiene a un enfoque que permita desarrollar el margen de maniobra y el poder de actuar de los actores (Coutarel, 2013).

10 En lo que concierne a los determinantes, éstos nos llevan a plantearnos el 'por qué' de la actividad. Un determinante representa un elemento de la situación de trabajo el cual nos ayuda a comprender el origen de la manera en la que el trabajador realiza su actividad, por lo tanto, la identificación de los determinantes es una parte importante de toda intervención para la modificación de las situaciones de trabajo (St-Vincent et al., 2011).

11 Es importante señalar que el artículo de Albert et al. (2017) señala que existe una diferencia entre las intervenciones realizadas en el terreno y las intervenciones realizadas en las investigaciones científicas en el plano de la prevención de los TME de origen laboral. En el primer caso, la intervención incluye diversas etapas (análisis de la demanda, análisis de las situaciones de trabajo, desarrollo de soluciones potenciales, desarrollo de prototipos, implementación y seguimiento) según las posibilidades y restricciones de la empresa, buscando comprender el trabajo real sin limitarse a las restricciones biomecánicas y buscando cambiar la representación de los actores claves respecto a las situaciones estudiadas, las causas de los problemas y las soluciones potenciales. En el segundo caso, los estudios se dirigen a un gran número de trabajadores de diferentes empresas a través de un ensayo controlado aleatorizado, abordándose de manera muy simplificada y reduciéndose a la solución implementada. Además, aquí resulta difícil saber si las diferentes etapas de una intervención han sido abordadas.

12 Existen diferentes modelos de estudio de TME que ayudan a los profesionales en Ergonomía para las intervenciones los cuales constituyen un soporte para establecer un referencial común entre los ergónomos y la diversidad de actores (incluso para los no especialistas de la salud en el trabajo) de la organización para facilitar la comprensión del problema de TME y apuntar hacia una prevención eficaz basada en los determinantes patógenos de las situaciones de trabajo y no en las 'malas prácticas' de los trabajadores (Buchmann \& Landry, 2010).

13 Cada modelo presenta aspectos diferentes y son complementarios, sin embargo, ayudan a construir la intervención y pueden adaptarse a cada contexto. El modelo de Franchi (1997) sitúa los factores de riesgo de los TME tales como los factores psicosociales, la 
sensibilidad individual y los factores biomecánicos alrededor de un conjunto de causas relacionadas a la organización, la producción, al ambiente de trabajo, al diseño de equipos, entre otros. El modelo de Bellemare, Marier, Montreuil, Allard \& Prévost (2002) propone poner en relación el análisis del trabajo con los síntomas o lesiones de los operadores por medio de las observaciones del trabajo real y entrevistas con diferentes actores para comprender los determinantes que conducen a la aparición de los factores de riesgo.

Finalmente, toda intervención ergonómica debe permitir algún tipo de transformación por medio de la co-construcción de soluciones con el fin de eliminar o reducir el riesgo. Estas soluciones se construyen colectivamente, conduciendo a la elaboración de soluciones detalladas por medio de interacciones entre el ergónomo y los actores de la empresa (St-Vincent et al., 2011).

\section{Objetivo}

presente intervención se enmarca dentro de la prevención de los riesgos laborales en la actividad de estiba de pescados congelados, buscando comprender los métodos y situaciones de trabajo que no están debidamente formalizados, proponiendo medidas que ayuden a prevenir lesiones, mejorando la productividad.

Para el análisis de las situaciones de trabajo, se hace necesario identificar los factores de riesgos a los cuales se exponen los estibadores y sus determinantes para plantear medidas adaptadas al contexto de la empresa en estudio.

\section{Metodología}

17 La metodología utilizada en el presente estudio está basada en el «análisis de la actividad» cuyo objetivo busca estudiar los modos operativos de los trabajadores en la situación real del trabajo (Guérin et al., 2007). A través de este análisis, se buscó comprender y reformular la demanda de la empresa y conocer su funcionamiento, describir la actividad de estiba de pescados congelados y analizar las situaciones de trabajo, no solo para buscar acciones correctivas sino también para cambiar la representación del trabajo al reducirlo a la manipulación de carga.

\section{Recojo de información}

El análisis de las actividades de estiba se ha llevado a cabo por medio de:

Análisis de información: se revisaron las matrices de identificación de peligros y evaluación de riesgo y los procedimientos de trabajo de las actividades de estiba.

Observaciones sistemáticas para comprender la distribución de las tareas y los modos operativos al realizar la colocación de los pescados congelados dentro de los contenedores por los estibadores. Además de analizar las posturas que adoptaban los estibadores al manipular la carga en los contenedores.

Entrevistas acerca del trabajo real a los estibadores en su mayoría con más de 10 años en el oficio con el objetivo de identificar los determinantes (o causas) que influyen en las situaciones del trabajo que pueden ocasionar daños a los estibadores o ser fuente de dificultades, interrogar sobre las molestias que han percibido en las diferentes zonas 
del cuerpo, determinar el nivel de esfuerzo percibido según la escala de Borg del 0 (nada) al 10 (muy muy duro), las dificultades que sienten durante su trabajo y las sugerencias para mejorar su puesto de trabajo. Por otro lado, se realizaron entrevistas a los supervisores de producción y supervisores de seguridad que se encontraban a cargo de las naves durante el turno con el objetivo de conocer más detalladamente los procesos.

22 Análisis de posturas de los distintos segmentos corporales a través del método REBA (Rapid Entire Body Assessment) al manipular los pescados congelados durante las observaciones. Según las puntuaciones obtenidas, este método clasifica el nivel de riesgo de la siguiente manera: inapreciable, bajo, medio, alto y muy alto (Hignett \& McAtamney, 2000). Si el nivel de riesgo es alto, se requiere la actuación cuanto antes o pronto, y si este nivel es muy alto, se requiera la actuación de inmediato.

El número de observaciones destinadas al caso de estiba de pescados congelados fue 4 días en diferentes turnos según el programa de atraque de los barcos. Cada turno dura 8 horas. Se eligió el turno mañana por temas de seguridad en el terminal portuario. Antes de cada observación, se reunieron a todos los integrantes de las cuadrillas para informarles sobre el objetivo del estudio de Ergonomía, la confidencialidad de los datos y solicitarles su participación y apertura para comprender el trabajo.

24 Las entrevistas fueron realizadas a los integrantes de las cuadrillas, conservando el anonimato, llevándose a cabo en su lugar de trabajo, cerca de los contenedores por periodos cortos cuando el trabajador no realizaba la estiba. Por ejemplo, durante el izaje de carga por la grúa.

Los cuestionarios de auto llenado no fueron empleados por la poca disponibilidad de tiempo de los estibadores y las restricciones de producción. No obstante, se mostró una imagen del cuerpo humano a los estibadores para señalar las partes del cuerpo donde han sentido molestias, y la tabla de la escala de Borg para señalar la intensidad del esfuerzo físico del trabajo según su percepción subjetiva. En este estudio, no se logró contar con un dinamómetro para realizar mediciones de la fuerza de arrastre al mover los pescados congelados.

\section{Descripción de trabajadores}

El mayor porcentaje de la población de los trabajadores portuarios pertenece a la especialidad de estibadores, gran parte de ellos tienen una edad mayor de 40 años según el registro de trabajadores portuarios del Ministerio de Transporte y Comunicaciones del año 2016. Menos del 50\% de los estibadores de la empresa es capaz de realizar la estiba de pescados congelados en ambientes de bajas temperaturas, dedicándose a estiba de otras cargas (ej. trinca y destrinca de contenedores, bolsones, sólido o líquido a granel).

Los trabajadores portuarios tienen diferentes especialidades tales como estibador, gruero, portalonero, elevadorista, tarjador según el Decreto Supremo Nº03-2013-TR. Todos los trabajadores portuarios deben encontrarse registrados por especialidad, acreditando capacitación o experiencia.

En el caso de estudio, la actividad de estiba de pescados congelados es realizada en cuadrillas de 10 a 12 estibadores bajo la supervisión de un capataz para ejecutar el plan de trabajo, ayudándose unos a otros en especial al manipular las cargas de gran tamaño. 
Todos los miembros de una cuadrilla (Caso A y B) fueron observados y entrevistados para comprender mejor la distribución de tareas.

La empresa cuenta con un sindicado de trabajadores portuarios. En este estudio, no se consideró si el estibador pertenecía o no al sindicato, ya que el sistema de rotación para el nombramiento de los estibadores y las cuadrillas para cada actividad es asumido por la misma empresa portuaria con la finalidad de evitar que personal sin experiencia realice diferente especialidad de carga y no afecte la productividad. Durante la intervención, no hubo ningún tipo de rechazo por parte de los estibadores en general.

\section{Descripción de los productos pesqueros}

El pescado congelado a granel como son los peces espadas, peces atún, aletas de tiburón y otros, pueden venir cubiertos en sacos para su conservación. Los tamaños y los pesos de estos productos son muy diversos, pudiendo exceder los $25 \mathrm{~kg}$ que es el peso máximo permitido para hombres por la normativa peruana Resolución Ministerial № $375-2008$. Cuando el pescado es muy grande y pesado (superior de $50 \mathrm{~kg}$ o $100 \mathrm{~kg}$ ), es frecuente que el traslado y el levantamiento de carga se realice entre dos o más personas

La figura 1 y 2 muestran los pescados que han sido extraídos de la bodega de la embarcación o nave por una grúa y luego es colocada dentro o fuera del contenedor. Durante el izaje de la carga, ninguna persona debe encontrarse debajo de la carga suspendida. Posteriormente, los estibadores deben apilarlo dentro del contenedor.

Figura 1. Pescados colocados dentro del contenedor por la grúa

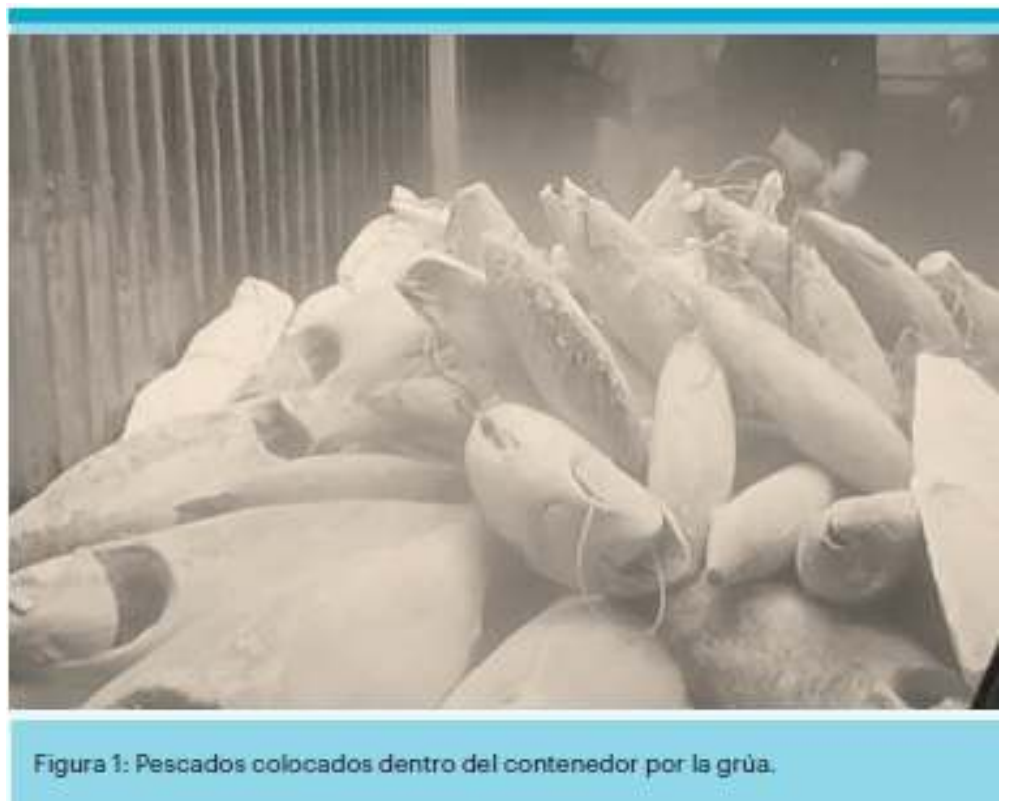


Figura 2. Pescados colocados cerca del contenedor por la grúa

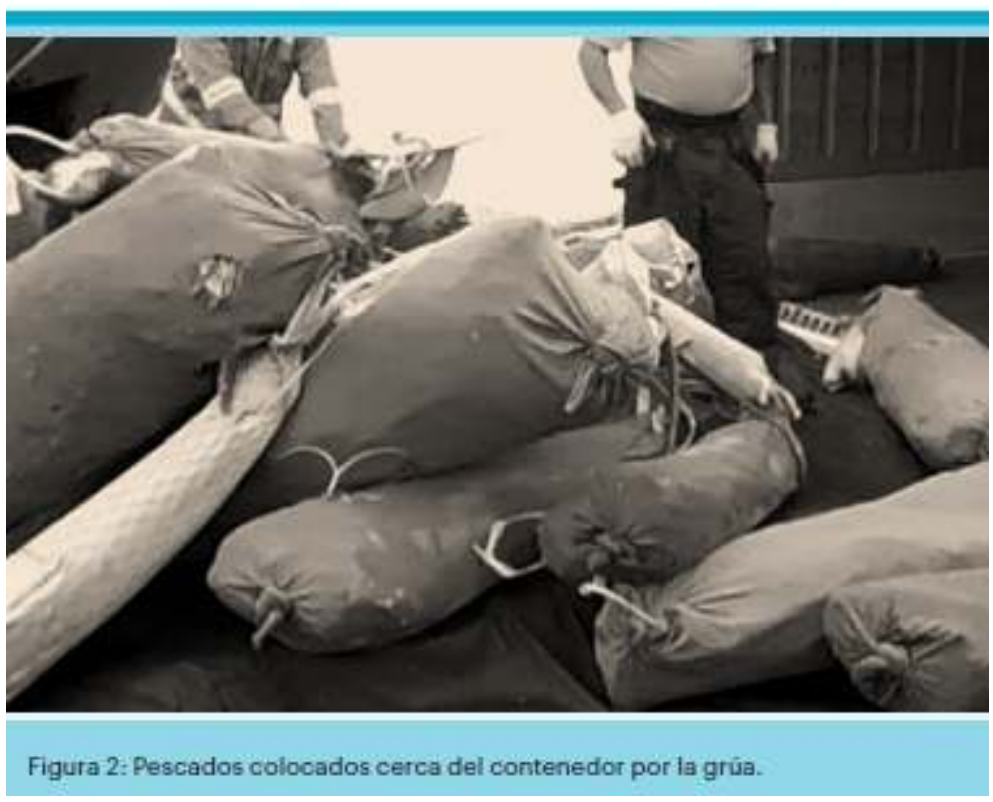

\section{Descripción de la ubicación de contenedores}

Para colocar la carga en un contenedor, se requiere por lo general 2 cuadrillas. La temperatura dentro de los contenedores Reefer puede llegar a temperaturas menores de $-10^{\circ} \mathrm{C}$. De manera estimada, el tiempo para colocar una carga de pescados congelados por los estibadores en el contenedor es de 5 a 10 minutos, dependiendo de la cantidad de pescados extraídos por la grúa. El tiempo de estadía promedio para las naves de productos pesqueros es alrededor de 34 horas.

La disposición de los contenedores puede ser de las siguientes maneras:

Caso A: Hay dos contenedores ( $\mathrm{C} 1$ y C2) uno al lado del otro, en este caso, encontramos una cuadrilla en cada contenedor. Primero, la carga es realizada en el contenedor C1 y luego, en el contenedor C2, y así se alternan.

Caso B: Hay un contenedor (C1) pegado junto a otro contenedor 'puerta a puerta', para impedir la entrada de aire a temperatura ambiente. En este caso, cada cuadrilla permanece la mitad del turno en los contenedores y la otra mitad es tomada como descanso, siendo la carga colocada por la grúa dentro del contenedor a través de una apertura en el techo. 
Figura 3 : Caso A

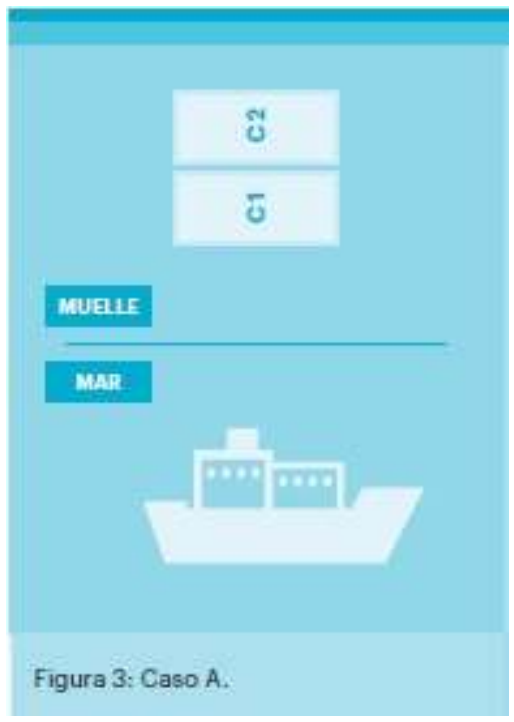

Figura 4 : Caso B

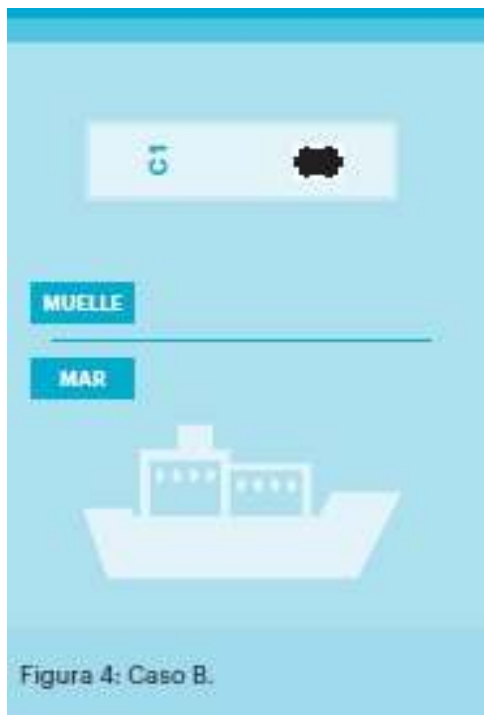

\section{Descripción de la distribución de tareas}

La carga extraída de las bodegas de las embarcaciones por la grúa puede contener pescados de diferentes tamaños. Los estibadores pueden distribuirse las tareas de las siguientes maneras:

\section{Caso de pescados pequeños:}

Alrededor de dos estibadores cogen los pescados colocados por la grúa cerca de la puerta del contenedor y pasan la carga a sus compañeros, quienes transportan los pescados dentro del contenedor, entregando la carga de un compañero a otro en las manos.

Alrededor de dos estibadores acomodan los pescados al fondo de la carga apilada dentro del contenedor. 
Caso de pescados grandes:

Cada estibador agarra uno o más pescados, utilizando un estrobo para jalar la carga.

Para levantar la carga, se requiere más de 5 estibadores dependiendo el peso del pescado.

Alrededor de dos estibadores acomodan la carga al fondo de la carga apilada sobre la ruma de pescados dentro del contenedor.

\section{Elección de Situaciones de trabajo a analizar}

La dificultad se presenta principalmente cuando los estibadores manipulan pescados grandes. En este artículo, se presentan las situaciones de trabajo relacionadas con la manipulación de estos productos que se da principalmente cuando los estibadores trasladan y apilan la carga en el contenedor. Durante la extracción de carga de las embarcaciones en el mar, el estibador en el muelle no realiza labor alguna.

Actividad: Carga de pescados congelados a granel

Situación $\mathrm{N}^{\circ} 1$ : Traslado de pescados congelados de gran tamaño con estrobo

El estibador utiliza un estrobo para entrelazarlo en la cuerda o cabo del pescado, y así poder trasladar la carga hasta el fondo del contenedor. También se observó que el estibador puede mover el pescado con la cuerda o cabo del pescado.

Situación $\mathrm{N}^{\circ} 2$ : Apilamiento de pescados congelados de gran tamaño por un grupo de personas

Para levantar un pescado de gran tamaño y peso excesivo desde el suelo del contenedor hasta el nivel alto de la carga apilada, los estrobos son colocados debajo del pescado para levantarlo simultáneamente por un grupo de estibadores.

\section{Situación $\mathrm{N}^{\circ}$ 3: Acomodo de pescados congelados de gran tamaño en el contenedor}

Encima de la carga apilada dentro del contenedor, encontramos dos estibadores de baja estatura que acomodan la carga sobre la ruma.

\section{Análisis de la demanda de la empresa}

52 El área de Seguridad y Salud en el Trabajo (SST) de la empresa en estudio de origen extranjero es consciente que el trabajo manual realizado por los estibadores los expone a una serie de factores de riesgo susceptibles de ocasionar daños a la salud, en especial, los relacionados a TME. Por tal razón, su requerimiento parte por la necesidad de mejorar los procesos de trabajo al no ser específicos en las buenas prácticas de manipulación manual de cargas (MMC). En este contexto, planteamos realizar un análisis de la actividad para una mejor comprensión del trabajo e identificar los diferentes factores de riesgo y sus determinantes en la estiba de pescados congelados, ya que es conocido que la formación en las buenas prácticas de MMC no es suficiente para solucionar el problema, si no tenemos en cuenta todo el contexto.

Cabe señalar que la empresa ha implementado equipos costosos en diferentes procesos de estiba, las cuales no han dado los resultados esperados ya sea por cuestiones de carácter operativo, de mantenimiento y/o humano. El personal no utiliza adecuadamente estos recursos por falta de capacitación, adiestramiento e 
insatisfacción, haciendo que las operaciones de producción se desarrollen con menos activos ya que ciertos equipos comienzan a fallar. Por lo tanto, el área de mantenimiento tiene que reparar con cierta frecuencia los equipos. En ocasiones, modificando sus características de diseño para su mejor uso, pero generando retrasos. Se constató que las iniciativas y sugerencias dadas por los estibadores no son tomadas en cuenta para su implementación.

\section{Resultados}

\subsection{Factores de riesgo}

La figura 5 muestra los factores de riesgo susceptibles de ocasionar daños a la salud y afectar el desempeño del personal en la actividad de estiba de pescados congelados para el caso estudiado.

Figura 5. Factores de riesgo en la estiba de pescados congelados

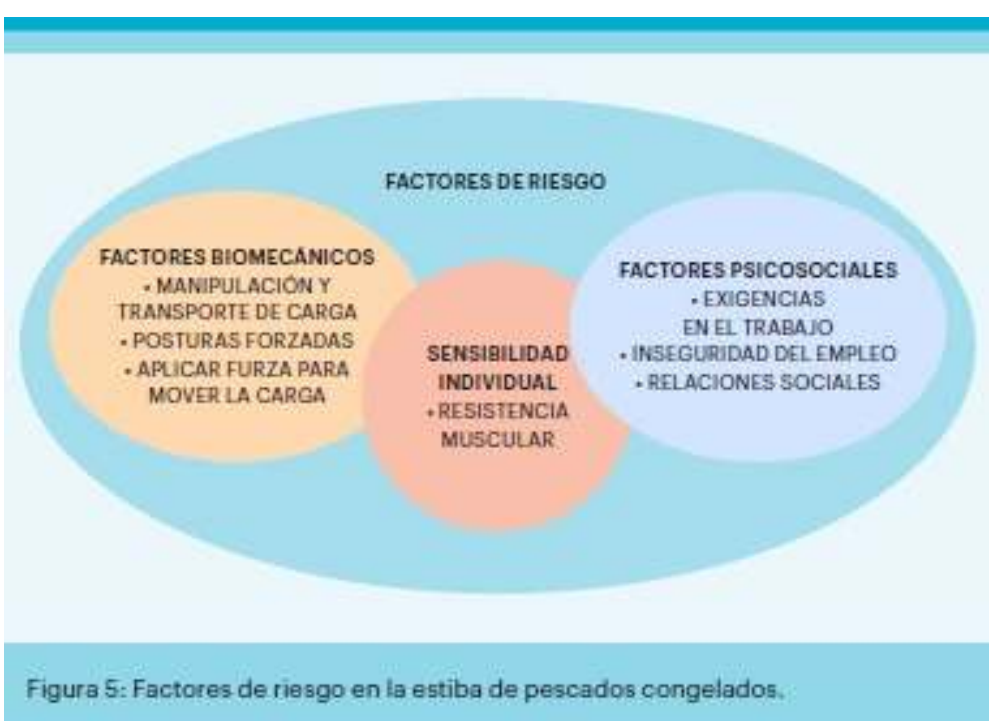

55 La estiba de pescados congelados se caracteriza por una excesiva carga física al manipular los pescados congelados de diferentes pesos y tamaños en ambientes de bajas temperaturas durante el turno de trabajo de 8 horas. El análisis de posturas a través del método REBA demostró que el trabajador está expuesto a posturas forzadas del tronco (flexión, extensión y torsión del tronco) y extremidades superiores (brazos alejados del cuerpo) al movilizar la carga con el estrobo. Asimismo, los estibadores deben aplicar fuerza para mover la carga con el estrobo (ej. >100kg) dentro de los contenedores cuyo piso es acanalado y se encuentra húmedo a una distancia máxima de recorrido de 12 metros dependiendo del largo del contenedor. El nivel de esfuerzo físico percibido al manipular los pescados de gran tamaño por todos los miembros de una cuadrilla de estibadores varía entre 8 y 10 según la escala de Borg, refiriéndose no solo por el peso y tamaño de la carga, sino también por el frío que sienten dentro del contenedor.

En cuanto a los factores individuales, la mayoría de estibadores han adquirido habilidades y destrezas, habituándose y tomando con naturalidad las exigencias físicas 
que se requieren para el desarrollo de las diferentes operaciones, caracterizándose por su resistencia física.

En relación a los factores psicosociales, los estibadores portuarios han presentado reclamos y han desarrollado huelgas en búsqueda de mejores condiciones de trabajo, mayores beneficios laborales y un mejor sistema de registro. Durante la intervención, no se constató espacios para la transmisión de ideas, sugerencias, con una política de reconocimiento e incentivos no bien precisados, especialmente cuando hay cumplimiento de metas de producción. Otro punto a tener en cuenta es la inseguridad del empleo al ser el personal contratado a destajo dependiendo del flujo de las embarcaciones durante todo el año. Por ley, el trabajador portuario puede trabajar 48 horas semanales y un máximo de 26 turnos efectivos por mes.

\subsection{Determinantes de las situaciones estudiadas de trabajo}

En esta parte, se busca identificar los principales determinantes (o causas) que influyen en las situaciones de trabajo por medio del diagrama de pescado o diagrama causaefecto. Las causas están representadas por las espinas del pescado unidas a su columna vertebral y a la cabeza del pescado (Freivalds \& Niebel, 2014). Las principales causas han sido subdivididas en categorías principales tales como producto, método de trabajo, medición, ambiente de trabajo, organización, individuo, equipos. producción por parte de los estibadores. Asimismo, evitar órdenes contradictorias entre seguridad y producción con el objetivo de proteger su capital humano y tener en cuenta las dificultades del trabajo para una mejor planificación de las tareas por el personal administrativo. Un aspecto a tener en cuenta es el origen de la empresa concesionaria del puerto cuya matriz no tiene el español como idioma oficial, pudiendo conllevar a problemas de comunicación con el mando operativo. 
Figura 6. Jalando los pescados con estrobo

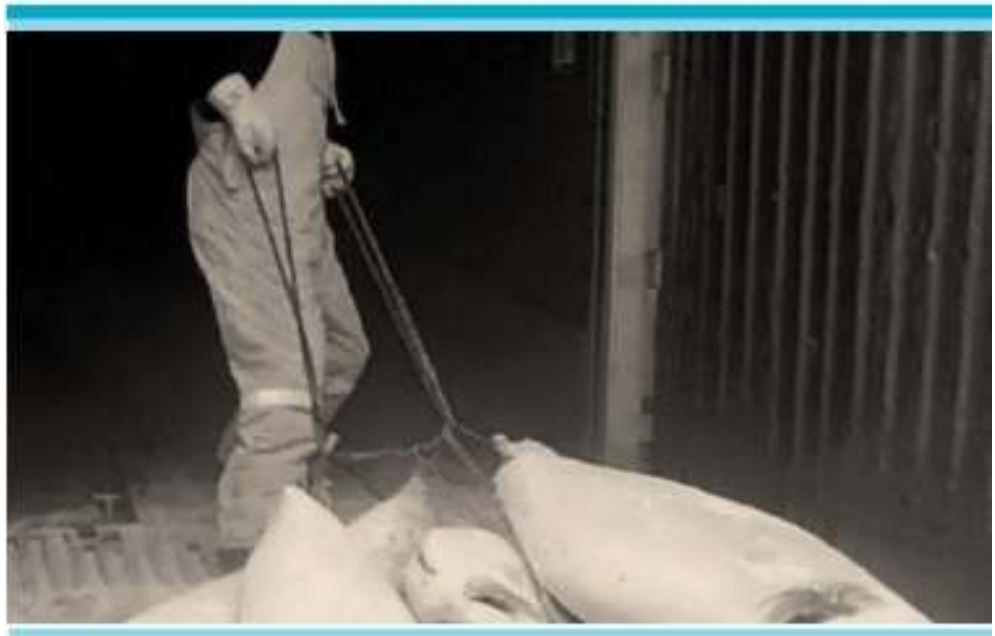

Figura 6: Jalando los pescados con estrobo.

Figura 7. Jalando los pescados sin estrobo

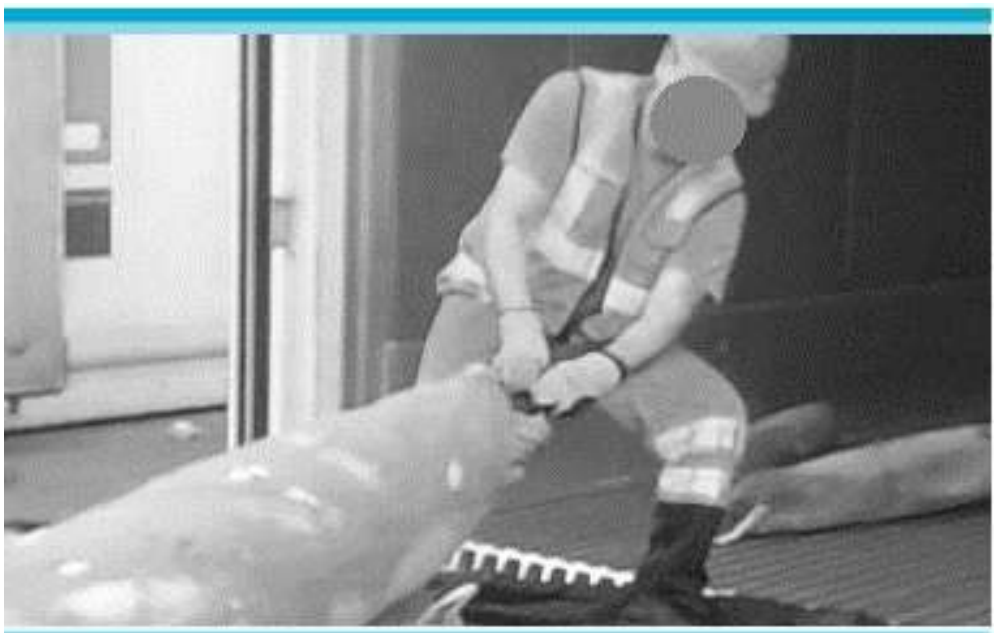

Figura 7: Jalando los pescados con estrobo. 


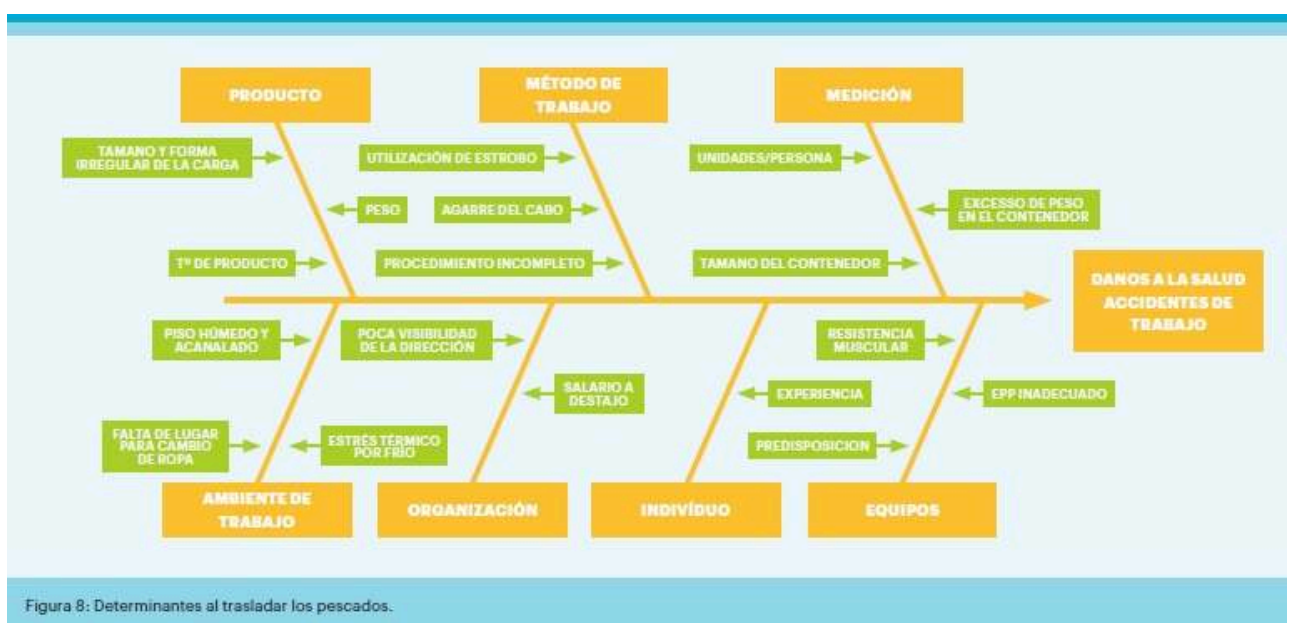

Situación $\mathrm{N}^{\circ} 2$ : Apilamiento de pescados congelados de gran tamaño por un grupo de personas

Cuando la carga es pesada y grande, se requiere levantarla entre más de 5 estibadores. La técnica consiste en colocar el estrobo debajo del pescado o en sostener alguna de las aperturas del cuerpo del pescado que han sido producidas en el proceso de evisceración y levantar la carga simultáneamente con ambas manos hasta introducirlo completamente sobre la posición donde se apilará.

La figura 9 resume los principales determinantes que influyen al apilar los pescados congelados en equipo. Se detectó la diferencia de estaturas entre los estibadores y la falta de un líder en el equipo durante el levantamiento de los pescados lo cual conlleva a una sobrecarga de trabajo en ciertos estibadores, aumentando el riesgo de lesión. Si la altura de la ruma de apilado es más alta $(>1 \mathrm{~m})$ respecto al suelo, se requiere de casi toda la cuadrilla para levantar el pescado congelado.

En esta situación, una mala repartición de las tareas y una mala comunicación puede conllevar a accidentes graves tales como golpes o resbalones al encontrarse el piso húmedo y al posible desprendimiento de los pescados apilados. Existe compañerismo entre los miembros de una cuadrilla a pesar de cometerse algún error, cada miembro tiene claro que el trabajo es en equipo. 


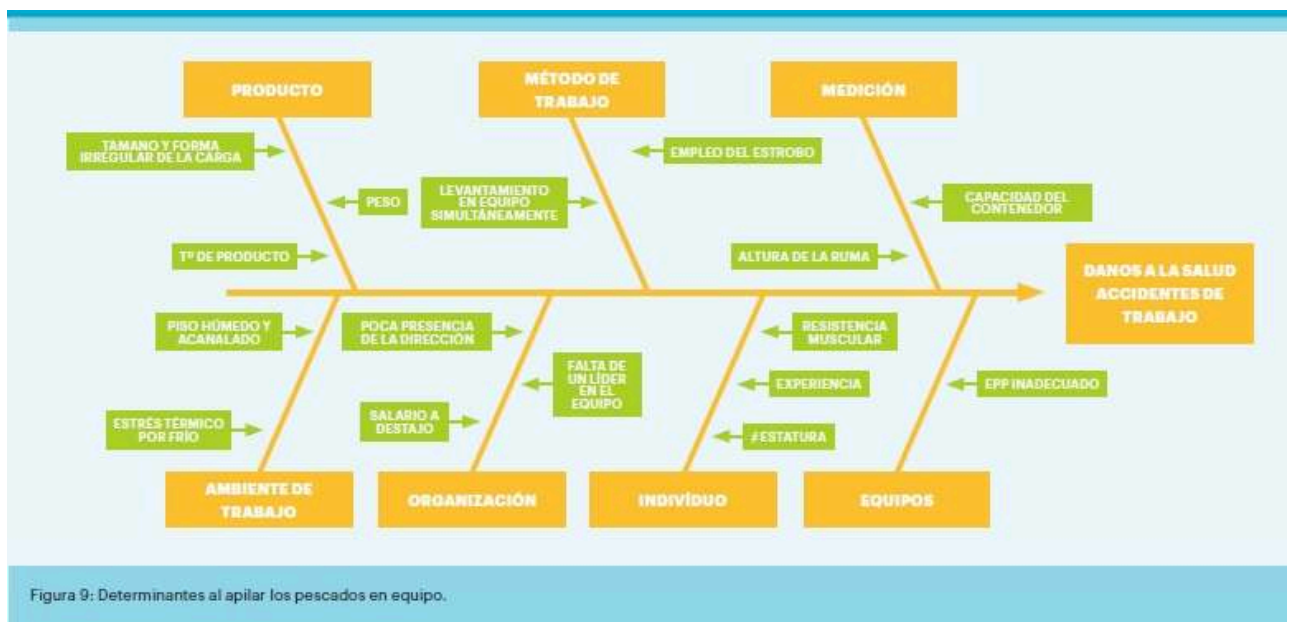

Situación $\mathrm{N}^{\circ} 3$ : Acomodo de pescados congelados de gran tamaño en el contenedor

Cuando se realiza la carga de dos contenedores, uno al lado del otro, encontramos dos estibadores de baja estatura posicionados sobre la ruma de los productos apilados para la recepción y el acomodo de la carga como se muestra en la figura 10. Se constató que estos estibadores adoptan posturas forzadas con flexión de espalda y piernas de forma permanente a causa del espacio reducido, y aplican fuerza para jalar y levantar el pescado.

En esta situación, el nivel de riesgo de la postura adoptada es muy alto según el método REBA. La figura 11 resume los principales determinantes que influyen al acomodar la carga arriba de la ruma. Aquí, los trabajadores de baja estatura no alternan de tareas durante su turno, exponiéndose a posturas forzadas en espacios reducidos e inestables por tiempo prolongado. La altura de apilado depende de las ordenes por parte de la tripulación. Si se detecta un exceso en la cantidad de carga dentro del contenedor en la zona de balanza, los estibadores deben retirar la carga y colocarla en otro contenedor.

Figura 10. Acomodo de carga arriba de la ruma

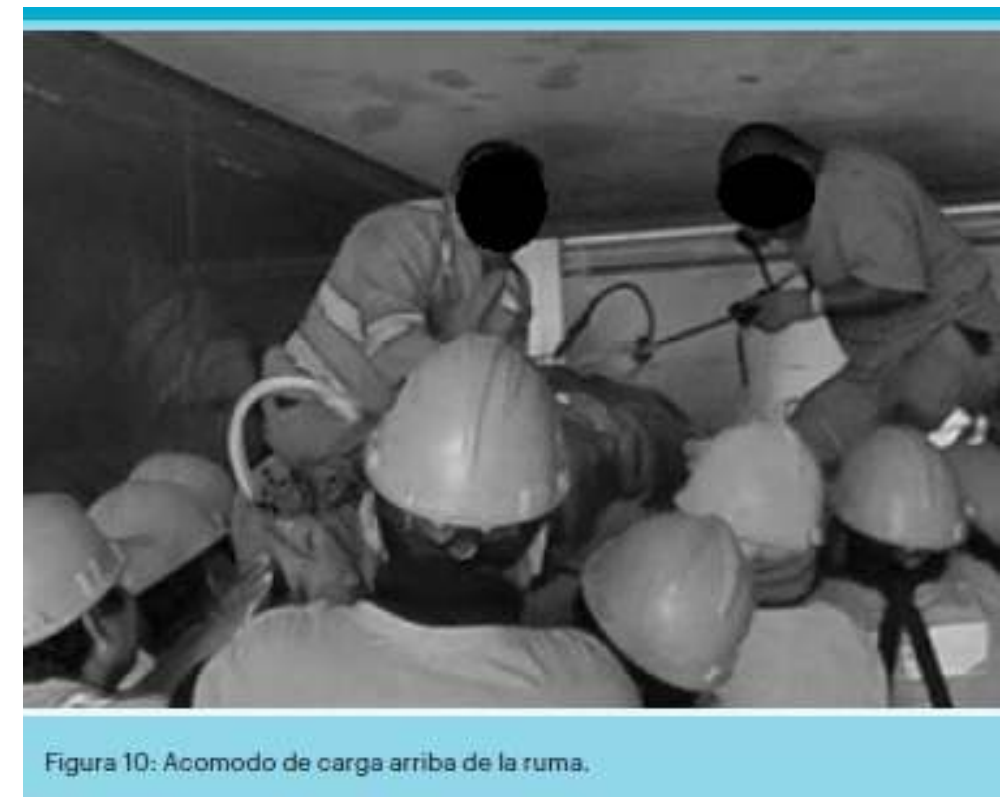


Figura 11. Determinantes al acomodar la carga

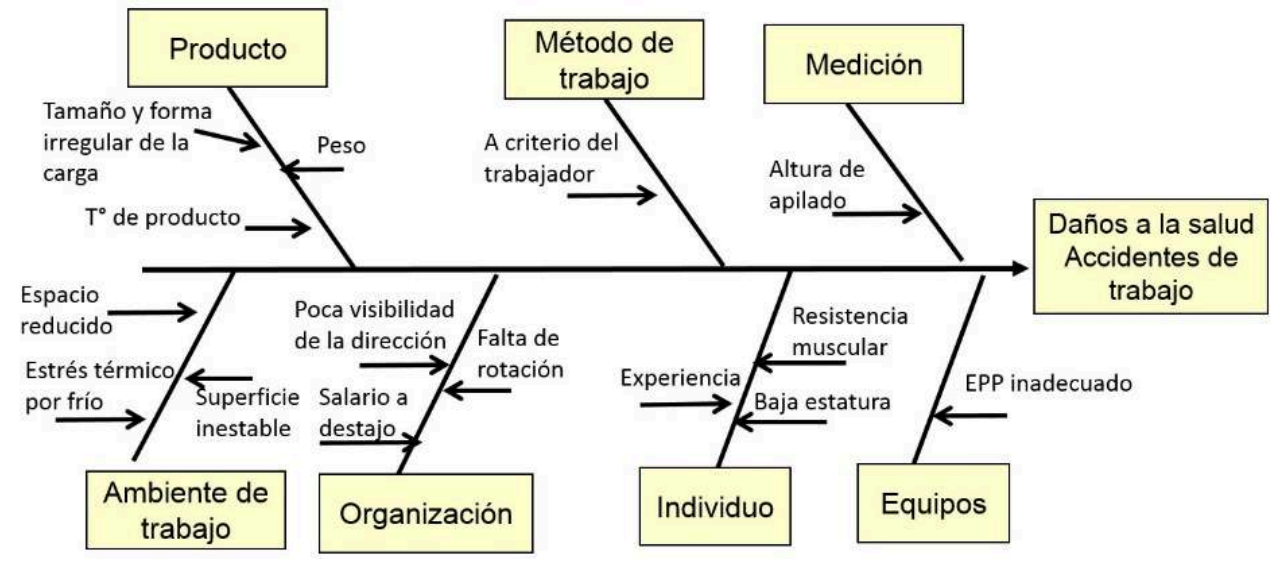

\subsection{Prácticas de regulación} problemas músculo-esqueléticos y lesiones corporales ocasionados por accidentes de trabajo. Los trabajadores señalaron haber sentido molestias principalmente a nivel de la zona lumbar, además de manos, brazos, rodillas y pies, cuando realizan la estiba de pescados congelados. Existe cierto temor por parte del personal en caso de detectarse alguna patología en el examen médico ocupacional, ya que, al no tener un contrato estable, correría el riesgo de no ser convocado para laborar. 
76 En cuanto a la producción, la empresa está sujeta a multas en caso de retrasos en la entrega de los contenedores llenos, presentándose un trabajo adicional en caso de excederse el peso permitido por contenedor, ya que tiene que retirarse parte de la carga para ser colocado en otro contenedor, aumentando la carga física de trabajo y probable daño de la mercancía.

77 Cuando la empresa no atiende oportunamente los reclamos relacionados a las condiciones y beneficios laborales de los estibadores con el fin de llegar a un acuerdo por medio de negociaciones colectivas, el tiempo de huelga (semanas) puede ocasionar millones de dólares en perdida.

\section{Discusión}

Durante la intervención, se buscó la participación de los trabajadores, supervisores de producción, supervisores y responsable de seguridad y salud en el trabajo, médico ocupacional y personal de mantenimiento. Sin embargo, hubo limitaciones para involucrar a la alta dirección y a otras áreas como las de procesos, recursos humanos y planificación. El artículo de Burgess-Limerick (2018) menciona que, para llevar a cabo un programa de ergonomía participativa, se requiere el compromiso de la gerencia a fin de poner a disposición los recursos (incluido tiempo para los miembros del equipo) y asegurar la implementación de medidas en la organización, sin embargo, una empresa con una cultura de desconfianza sin algún antecedente de participación de los trabajadores en la toma decisión, es poco probable que la ergonomía participativa prospere.

La Ley $N^{\circ} 29783$ de Seguridad y Salud en el Trabajo exige a las empresas peruanas realizar estudios de ergonomía. Es frecuente que algunos actores del área de seguridad y salud en el trabajo reduzcan la contribución de la ergonomía a la prevención de los TME 'si no hay dolor, no se hace ergonomía'. Por lo tanto, el profesional de ergonomía debe jugar un rol activo para cambiar la representación de los actores y ayudar a la empresa a crear espacios de intercambio donde el personal pueda expresar sus propuestas y dificultades.

El análisis de la actividad permite sacar a la luz que los estibadores adoptan posturas forzadas y realizan esfuerzos para mover o levantar los pescados congelados en ambientes de bajas temperaturas, buscando cumplir con las metas de producción con un margen de maniobra reducido para intervenir en la forma en que se planifica el trabajo. En una siguiente intervención, resulta necesario medir la fuerza de inicio y de sustentación para establecer el peso límite al jalar la carga.

81 La Resolución Ministerial N³75-2008 Norma Básica de Ergonomía y de Procedimiento de Evaluación de Riesgo Disergonómico recomienda no exceder el peso de $25 \mathrm{~kg}$ para la MMC para hombres, pero no señala la postura segura (ej. carga cerca del cuerpo, espalda derecha sin flexiones y rotaciones) y no considera para el caso de la temperatura baja del ambiente en las tareas de manipulación. Esta normativa también permite $40 \mathrm{~kg}$ para trabajadores entrenados y/o situaciones aisladas, y puede aceptarse también para tareas esporádicas y trabajadores sanos. Es necesario señalar que, en el Perú, no existe una reglamentación en material psicosocial a fin de contribuir al desarrollo de organizaciones saludables. 


\section{Conclusiones} eficiencia en el tiempo de estadía de los buques en el puerto y mejorar la fluidez de las operaciones de carga y descarga ha ido adquiriendo nuevos equipos (ej. reach stackers o manipulador de contenedores). En el caso de los productos pesqueros, el trasbordo de pescado a granel de un barco pesquero a un contenedor que demora más de un día, se requiere que los productos sean colocados sobre la plataforma del contenedor al ser una actividad manual, debido a la variedad de tamaño y forma de los pescados. El empleo de ayudas mecánicas que ayuden a movilizar los pescados congelados de gran tamaño para consumo humano dentro de contenedores sigue siendo un reto para reducir el riesgo sin dañar y contaminar el producto. Al existir una gran variabilidad de posturas dinámicas debido a la diversidad de pescados y la presencia de posturas estáticas en los apiladores de carga localizados al fondo de los contenedores, el uso de exoesqueletos es aún un reto para adaptarse a esta tarea y aún no se encuentra dentro de los planes de inversión de la empresa.

Por lo tanto, al ser un trabajo manual, se requiere que la organización garantice un adecuado sistema de registro de sus trabajadores portuarios para una mejor selección de los estibadores, distribución de los turnos de trabajo e implementar una política de reconocimiento e incentivos por cumplimiento de metas de producción. Para una mejor atención de las solicitudes de los estibadores, la empresa debe crear espacios de negociación por medio de sus representantes para tomar en cuenta sus sugerencias propuestas que tienen relación con la mejora del servicio que se da a los clientes. Para 
ello, se requiere el involucramiento de la alta dirección en el lugar de trabajo para escuchar y conocer la realidad del trabajo.

El trabajo en cuadrilla debe permitir una buena coordinación y distribución de la carga física de trabajo entre los miembros, con una adecuada selección de personal, para no producir una sobrecarga en algunos estibadores. Se elaboró un Manual de Actividades dirigido a estibadores y supervisores, ya que los procedimientos no señalaban, de manera específica, la forma de manipular los pescados congelados pequeños y grandes. Se requiere formar líderes para un mejor control al manipular la carga para evitar entorpecer los movimientos. La técnica de utilizar los estrobos reduce las flexiones excesivas del tronco al evitar jalar la carga cerca del nivel del piso. Sin embargo, se deben evitar sacudidas bruscas y jalar varios pescados de gran tamaño a la vez.

Por otro lado, la empresa debe proporcionar los equipos de protección personal contra el frío para evitar el entumecimiento de los músculos, los cuales deben ser de uso personal y adaptado a la medida, además de acondicionamientos sencillos para brindar un mejor confort (ej. ambiente adecuado para el cambio de vestimenta, consumo de bebidas calientes). Para reducir el cansancio y el desgaste muscular por la exposición a bajas temperaturas, se sugirió rotar los turnos siguientes a labores de estiba de otras cargas de menor exigencia física (ej. sólido o líquido a granel). Además, la empresa debe garantizar un adecuado sistema de vigilancia médica ocupacional.

Al realizar el cambio de turno, se propuso que los estibadores del turno saliente sean reunidos para brindar retroalimentación sobre los problemas encontrados a los estibadores del turno que ingresa, involucrando al personal de producción al supervisar las embarcaciones. Asimismo, la organización debe empoderar a los supervisores de SST para detener la producción en caso de encontrarse fallas técnicas y/o organizacionales en el desarrollo del trabajo que tengan el potencial de ocasionar accidentes graves o fatales.

Finalmente, futuras investigaciones deberían permitir una mejor explicación de los diversos trabajos portuarios en el Perú y América Latina sin limitarse a la valoración del nivel del riesgo y buscar involucrar a los diferentes actores de la organización teniendo en cuenta su cultura laboral para favorecer la resolución de problemas.

\section{BIBLIOGRAFÍA}

Albert, V., Vézina, N., Bilodeau, H. \& Coutarel, F. (2017). Comment et pourquoi: proposition des processus de la phase de développement d'intervention ergonomiques. Perspectives interdisciplinaires sur le travail et la santé, 19-3. https://journals.openedition.org/pistes/5513

Bellemare, M., Marier, M., Montreuil, S., Allard, D. \& Prévost, J. (2002). La transformation des situations de travail par une approche participative en ergonomie : une recherche intervention pour la prévention des troubles musculo-squelettiques. Études et recherches, Rapport R-292. Montréal: IRSST. http://www.irsst.qc.ca/fr/_publicationirsst_824.html 
Buchmann, W., \& Landry, A. (2010). Intervenir sur les TMS. Un modèle des Troubles Musculosquelettiques comme objet intermédiaire entre ergonomes et acteurs de l'entreprise. Activités 7(2), 84-103. https://journals.openedition.org/activites/2418

Bourgeois, F., \& De Gasparo, S. (2011). La privation d'agir, objet commun d'analyse des TPS et TMS. In F. Hubault (coord.). Risques psychosociaux: quelle realité, quells enjeux pour le travail (pp. 85-99). Toulouse: Octarès Éditions.

Bourgeois, F., Lemarchand, C., Hubault, F., Brun, C., Polin, A., Faucheux J.-M. ... Albert, E. (2006). Troubles musculosquelettiques et travail. Quand la santé interroge l'organisation. Lyon: ANACT.

Burgess-Limerick, R. (2018). Participatory ergonomics: evidence and implementation lessons. Applied Ergonomics, 68, 289-293.

Coutarel, F., \& Petit, J. (2013). Prévention des TMS et développement du pouvoir d'agir. In P. Falzon (dir), Ergonomie constructive, (pp. 175-190). Paris: PUF.

Franchi, P. (1997). Les maladies professionnelles: l'exemple des troubles musculosquelettiques (TMS). Lyon: ANACT.

Freivalds A., \& Niebel, B. W. (2014). Ingeniería industrial de Niebel métodos, estándares y diseño del trabajo. México: McGraw-Hill/Interamericana Editores.

Garg, A., Boda, S., Hegmann, K. T., Moore, J. S., Kapellusch, J. M, Bhoyar, P. Malloy, E. J. (2014). The NIOSH Lifting Equation and Low-Back Pain, Part 1 Association With Low-Back Pain in the BackWorks Prospective Cohort Study. Human Factors, 56, 6-28. http://journals.sagepub.com/doi/ pdf/10.1177/0018720813486669

Guérin, F., Laville, A., Daniellou, F., Duraffourg, J. \& Kerguelen, A. (2007). Comprendre le travail pour le transformer. La pratique de l'ergonomie. Lyon: ANACT.

Hignett, S., \& McAtamney, L. (2000). Rapid Entire Body Assessment (REBA). Applied Ergonomics, 31(2), 201-205.

Monroy, E. M., \& Luna, P. M. (2015). NTP 1.036 Estrés por frío (I). Instituto Nacional de Seguridad e Higiene en el Trabajo. España.

St- Vincent, M., Vézina, N., Bellemare, M., Denis, D., Ledoux, É. \& Imbeau, D. (2011). L'intervention en ergonomie. Québec: Éditions MultiMondes.

\section{RESÚMENES}

En los terminales portuarios del Perú, la colocación dentro de contenedores refrigerados de productos pesqueros tales como los pescados congelados se realiza manualmente con la ayuda de estrobos. Los trabajadores son organizados en cuadrillas según sus experiencias y conocimientos con el objetivo de cumplir con las metas de producción, respetando las normas de calidad y seguridad señaladas por la organización. La introducción de tecnologías en las actividades de estiba de carga no han tenido los resultados esperados en la reducción de la exigencias físicas a largo plazo en el personal operativo, quienes poseen los conocimientos de los procesos, representando una desvalorización de la experiencia y una pérdida de dinero en el recurso tecnológico al ser inutilizado por los propios trabajadores. Esta intervención ergonómica tiene por reto analizar la estiba de pescados congelados bajo un enfoque sistémico para comprender las formas de trabajo y los determinantes, involucrando a los estibadores. 
Nos terminais portuários do Perú, a colocação de peixe congelado no interior dos contentores refrigerados é efetuado manualmente com a ajuda de cabos. Os trabalhadores são organizados em grupos em função da sua experiência e conhecimentos a fim de responderem a objetivos de produção, respeitando as normas de qualidade e de segurança prescritas pela organização. Neste contexto a introdução de tecnologias nas atividades de manutenção não teve os resultados esperados em termos de redução das exigências físicas de longo prazo com os trabalhadores que possuem o conhecimento dos processos. Isto que significa uma desvalorização da experiência e uma perda de rentabilidade no investimento num recurso tecnológico não utilizado. Esta intervenção ergonómica teve como desafio analisar o trabalho da manutenção de peixe congelado respeitando uma abordagem sistémica a fim de compreender as formas do trabalho e os seus determinantes com a participação dos trabalhadores.

Dans les terminaux portuaires du Pérou, le chargement des poissons congelés à l'intérieur des conteneurs réfrigérés, est effectué manuellement à l'aide d'un cordage. Les travailleurs sont organisés en groupes en fonction de leur expérience et connaissances afin de répondre aux objectifs de production, en respectant les normes de qualité et de sécurité prescrites par l'organisation. Dans ce contexte, l'introduction de technologies dans les activités de manutention n'a pas eu les résultats escomptés en termes de réduction des contraintes physiques à long terme chez les travailleurs qui possèdent la connaissance des processus, ce qui représente une dévaluation de l'expérience et une perte d'argent investi dans une ressource technologique non utilisée par eux. L'intervention ergonomique a pour défi d'analyser le travail lié à la manutention de poissons congelés suivant une approche systémique afin de comprendre les formes de travail et les déterminants, avec la participation des travailleurs.

In the port terminals of Peru, the placement of fishery products such as frozen fish within refrigerated containers is done manually with the help of gaff hooks. The workers are organized into groups according to their experiences and knowledge in order to meet the production goals, following the quality and safety standards indicated by the organization. The introduction of technologies in the process of cargo stowage did not meet the expected results on reducing the long-term physical effects on operational personnel, who are the ones with the knowledge for the processes, which represents a disregard of the experience and loss of money in technology that is wrongly used by the workers. The challenge of this ergonomic intervention is to analyze the process of cargo stowage of frozen fish under a systematic approach to understand the ways and factors of this job, while getting the workers involved.

\section{ÍNDICE}

Mots-clés: travail manuel, arrimage, facteurs de risque, déterminants

Palavras-chave: trabalho manual, estiva, fatores de risco, determinantes

Keywords: manual work, stowage, risk factors, causes

Palabras claves: trabajo manual, estiba, factores de riesgo, determinantes

\section{AUTORES}

\section{CAROLINA ULLILEN-MARCILLA}

Facultad de Ingeniería Ambiental, Escuela Profesional de Ingeniería de Higiene y Seguridad Industrial, Universidad Nacional de Ingeniería, Av. Túpac Amaru 210, Rímac. Lima 21, Perú. cullilenm@uni.edu.pe 


\section{ROMEL ULLILEN-MARCILLA}

Facultad de Ciencias, Licenciado en Matemática, Universidad Nacional de Ingeniería, Av. Túpac Amaru 210, Rímac. Lima 21, Perú.

romel.ullilen.marcilla@gmail.com 